\title{
INTROGRESIÓN DE LOS GENES DE RESISTENCIA A ROYA AMARILLA Yr5a Y Yr15 EN EL CULTIVAR DE TRIGO HARINERO BORLAUG 100
}

\author{
INTROGRESSION OF Yr5a AND Yr15 YELLOW RUST RESISTANCE \\ GENES INTO THE BORLAUG 100 BREAD WHEAT CULTIVAR
}

\author{
Yerica R. Valdez-Rodríguez ${ }^{1}$, Julio Huerta-Espino ${ }^{2} \star$, J. Sergio Sandoval-Islas ${ }^{1}$, \\ Héctor E. Villaseñor-Mir ${ }^{2}$ y Olga Gómez-Rodríguez ${ }^{1}$
}

\begin{abstract}
'Colegio de Postgraduados, Campus Montecillo, Programa de Fitopatología, Montecillo, Texcoco, Estado de México, México. ${ }^{2}$ Instituto Nacional de Investigaciones Forestales, Agrícolas y Pecuarias, Campo Experimental Valle de México, Coatlinchán, Texcoco, Estado de México, México.

*Autor de correspondencia (j.huerta@cgiar.org)
\end{abstract}

\section{RESUMEN}

La producción de trigo (Triticum aestivum L.) es afectada por diversos factores bióticos como la roya amarilla causada por Puccinia striiformis f. sp. tritici. Este patógeno tiene el potencial de ocasionar pérdidas en el rendimiento de hasta $100 \%$ si los cultivares susceptibles son infectados en etapas tempranas de crecimiento. La mejor estrategia para el manejo de esta enfermedad es el uso de genotipos resistentes. El objetivo del presente estudio fue transferir los genes Yr5a y Yr15 de Blanca Grande 515 al cultivar Borlaug 100 (parental recurrente) y obtener líneas resistentes manteniendo las características agronómicas de la misma. Se obtuvieron 389 líneas a partir de dos y tres retrocruzas entre Borlaug 100 y Blanca Grande 515, las cuales se evaluaron en plántula y en planta adulta en campo contra el aislado CMEX14.25. La presencia de los genes se corroboró con marcadores moleculares SNP. Los resultados indicaron que Yr5a, previamente reportado en estado homocigótico en Blanca Grande 515, en realidad se encuentra en forma heterocigótica, por lo que el número de líneas positivas a este gen fue bajo en comparación con las positivas para Yr15. El número de combinaciones de genes encontradas fue menor que lo esperado y sólo se encontraron líneas positivas para los dos genes de interés después de realizar la segunda retrocruza. Del total de líneas generadas, se identificaron tres que poseen los genes Yr5a y Yr15 en condición homocigótica, una semejante a Blanca Grande 515 y otra con ambos genes en condición heterocigótica. Dos de las tres primeras líneas también poseen el gen Yr17, proveniente de Borlaug 100, y fenotípicamente fueron muy semejantes a ese cultivar, lo que demuestra que el método de retrocruzamiento es efectivo para transferir y acumular mayor cantidad de genes para lograr resistencia durable manteniendo el fenotipo del progenitor recurrente.

Palabras clave: Triticum aestivum, MAS, resistencia genética, roya amarilla.

\section{SUMMARY}

Wheat (Triticum aestivum L.) production is affected by various biotic factors such as yellow rust caused by Puccinia striiformis $\mathrm{f}$. sp. tritici. This pathogen has the potential to cause yield losses of up to $100 \%$ if susceptible cultivars are infected in early growth stages. The best strategy for the management of this disease is the use of resistant genotypes. The objective of this study was to transfer the Yr5a and Yr15 genes from the Blanca Grande 515 population to the Borlaug 100 cultivar (recurrent parent) to obtain resistant lines while maintaining its agronomic characteristics. Three hundred eighty-nine lines were obtained from two and three backcrosses between Borlaug 100 and
Blanca Grande 515, which were evaluated at the seedling stage and adult plant in the field against the CMEX14.25 isolate. The presence of the genes was corroborated with SNP molecular markers. Results indicated that Yr5a, previously reported in homozygous state in Blanca Grande 515, is actually heterozygous, so the number of positive lines for this gene was low compared to those positives for Yr15. The number of gene combinations found was lower than the expected and positive lines for the two genes of interest were found after the second backcross. Three lines were identified as having both $\mathrm{Yr} 5 \mathrm{a}$ and $\operatorname{Yr} 15$ genes in homozygous condition, one similar to Blanca Grande 515 and another with both genes in heterozygous condition. Two of the first three lines also possess the Yr17 gene, from Borlaug 100, and phenotypically they were very similar to that cultivar, which shows that the backcross method is effective in transferring and accumulating genes to achieve durable resistance while maintaining the phenotype of the recurrent parent.

Index words: Triticum aestivum, genetic resistance, MAS, yellow rust

\section{INTRODUCCIÓN}

La producción de trigo es afectada por factores bióticos y abióticos, dentro de los primeros se encuentran las enfermedades fungosas, principalmente las ocasionadas por especies del género Puccinia spp. La roya amarilla, causada por Puccinia striiformis f. sp. tritici, está presente en más de 60 países y, de acuerdo con la incidencia y severidad con la que se presente la enfermedad, puede ocasionar pérdida en el rendimiento hasta de $100 \%$ en variedades susceptibles infectadas en etapas tempranas (Begum et al., 2014; Wellings, 2011).

Se han identificado 78 genes (Yr) que confieren resistencia a las diferentes razas de roya amarilla (Mclntosh et al., 2017). El gen Yr5 fue descrito por Macer (1966) en Triticum spelta var. album, la resistencia de éste no se utilizó en cultivares comerciales de trigo por mucho tiempo después de su identificación, hasta hace poco Recientemente, Marchal et al. (2018) clonaron Yr5 (en adelante referido como Yr5a) y mostraron que es alélico a YrSP (Spalding Prolific) $=$ Yr5b y parálogo a Yr7. El gen Yr15 
se descubrió en la década de los 1980s en la accesión G25 de Triticum dicoccoides (Gerechter-Amitai et al., 1989). Desde su introgresión inicial en Israel, los programas de mejoramiento han utilizado las líneas resultantes como fuente de resistencia en cultivares de trigo duro y harinero (Chen y Kang, 2017).

En la última década se han reportado epidemias de roya amarilla en diferentes regiones del mundo, incluyendo el centro y oeste de Asia, norte y este de África (Ali et al., 2017), así como en Europa con la raza Warrior (virulenta a: Yr1, Yr2, Yr3, Yr4, Yr6, Yr7, Yr9, Yr17, Yr32 y Yr5b), que se identificó por primera vez en 2011 en el Reino Unido (Hubbard et al., 2015). Ali et al. (2017) llevaron a cabo un estudio durante 2009-2015 en el que recolectaron 887 aislados pertenecientes a 79 razas diferentes de 35 países. Yr5a y Yr15 fueron los únicos genes para los que no se observó virulencia, aunque Nagarajan et al. (1986) reportaron lo contrario para Yr5a en India y Australia y, posteriormente, Wellings et al. (2009) reportaron un aislado virulento en Australia. En la actualidad se considera que ninguna raza es virulenta a la combinación Yr5a y Yr15 (Ali et al., 2017; Sharma-Poudyal et al., 2013).

En México la roya amarilla está presente en todas las zonas productoras de trigo, hasta el año 2001 el aislado más común era MEX 96.11, virulento a Yr2, Yr3, Yr6, Yr7, Yr9 y Yr27 (Huerta-Espino et al., 2015). En 2003 una raza con virulencia a Yr8 ya se encontraba ampliamente distribuida y evolucionó venciendo la resistencia a Yr7. Para el año 2008 se detectaron variantes virulentas a Yr17 y Yr37 (HuertaEspino et al., 2015). Rodríguez et al. (2009) y RodríguezGarcía et al. (2010) demostraron virulencia para los genes Yr1, 2, 3, 6, 7, 8, 9, 17, 27, Poll y A en los Valles Altos de México y regiones productoras de trigo de riego. En 2014 se identificaron los aislados CMEX14.25 (CEVAMEX14.25) y MEX 14.141 virulentos a Yr2, Yr3, Yr6, Yr7, Yr8, Yr9, Yr77, Yr27 y Yr37, lo que ocasionó pérdidas en los rendimientos del cultivar Luminaria F2012, recomendado para siembras en áreas de riego en El Bajío (Solís et al., 2016), así como en el cultivar Nana F2007, recomendado para siembras de temporal en México (Villaseñor et al., 2014), en el que se registraron pérdidas en rendimiento de hasta $84 \%$ (Díaz et al., 2018). El patógeno continuó evolucionando y posteriormente se identificaron los aislados MEX16.03 y MEX16.04 con virulencia a Yr10 y Yr24, lo que dejó efectivos solamente a los genes Yr5a, Yr15 y Yr5b (HuertaEspino y Singh, 2017).

Ante estos hechos, es claro que el uso de cultivares resistentes es la mejor estrategia de manejo de la enfermedad, ya que no ocasiona costos adicionales para el agricultor por el uso de fungicidas y es segura para el medio ambiente (Singh et al., 2002). El método de retrocruzas es muy empleado por los programas de mejoramiento y es el más adecuado para transferir un gen (o pocos genes) a un cultivar adaptado, de modo que el único cambio en el cultivar elite se deba a los genes transferidos (Acquaah, 2012). Adicionalmente, es posible llevar a cabo selección asistida por marcadores (MAS) debido a que permite acelerar el mejoramiento tradicional al emplear marcadores moleculares y los genes deseables pueden fijarse en las primeras generaciones del programa de mejoramiento (Vagndorf et al., 2018).

Borlaug 100 (Bor100) es un cultivar de trigo harinero de hábito de crecimiento primaveral que se originó de la selección de poblaciones segregantes de la cruza ROELFS07/4/BOW/NKT//CBRD/3/CBRD/5/FRET2/ TUKURU//FRET2, llevada a cabo por el Programa de Trigo Harinero del Centro Internacional de Mejoramiento de Maíz y Trigo (CIMMYT), y de su posterior selección por el Programa de Mejoramiento Genético de Trigo del Instituto Nacional de Investigaciones Forestales, Agrícolas y Pecuarias (INIFAP) del Campo Experimental Norman E. Borlaug (Camacho et al., 2017). Bor100 posee el gen de resistencia Yr17 proveniente de Aegilops ventricosa y para el cual no hay virulencia total en las poblaciones actuales de roya amarilla. Bor100 en estado de plántula es casi inmune hasta moderadamente susceptible, dependiendo de la raza del patógeno; ante la raza MEX18.191 es susceptible en plántula y en planta adulta muestra infecciones de 40MR a 60MR en la escala del 0-100.

Blanca Grande 515 (BG515) se originó de la cruza BLANCA-GRANDE/5*Yr5_and_Yr15 (GRIS, 2017), es de hábito de crecimiento primaveral y se liberó por Resource Seeds, Inc. en 2010. Los genes de resistencia Yr5a y Yr15 se introdujeron por cuatro generaciones de retrocruzamiento en el cultivar susceptible Blanca Grande y luego se combinaron mediante selección asistida por marcadores en la Universidad de California, Davis (Jackson, 2011).

Tomando como base la durabilidad de la combinación Yr5a + Yr15 y la ausencia de virulencia a nivel mundial, el objetivo del presente estudio fue transferir los genes Yr5a y Yr15 al cultivar Borlaug 100 manteniendo las características agronómicas de la misma.

\section{MATERIALES Y MÉTODOS}

\section{Material genético}

Se utilizó el cultivar BG515 como progenitor donador y como progenitor recurrente a Bor100. Se emascularon espigas de Bor100 y se polinizaron con el cultivar BG515 de donde se derivó la $F_{1}$ que se retrocruzó hacia Bor100 para producir las semillas $\mathrm{BC}_{1}$. Se identificaron plantas positivas 
para el gen Yr15 mediante marcadores moleculares en la generación $\mathrm{BC}_{1} \mathrm{~F}_{1}$, mismas que se retrocruzaron hacia Bor100 para generar semillas $\mathrm{BC}_{2} \mathrm{~F}_{1}$ y se seleccionaron las positivas a Yr15 mediante el uso de marcadores moleculares. Posteriormente, las plantas seleccionadas se retrocruzaron hacia Bor 100 para generar semillas $\mathrm{BC}_{3} \mathrm{~F}_{1}$. En esta etapa de la retrocruza no se utilizaron marcadores para detectar Yr5a, ya que la mayoría de los marcadores previamente reportados no mostraron ser de diagnóstico cuando se probaron en diversas fuentes de germoplasma (Naruoka et al., 2016).

De la segunda retrocruza $\left(\mathrm{BC}_{2}\right)$ en la generación $F_{2^{\prime}}$ por un lado, se seleccionaron 135 plantas que fueron avanzadas hacia homocigosis a través de familias por surco para derivar 135 líneas $F_{6}$; por otro lado, también en la $F_{2}$ se seleccionó un número indefinido de plantas que fueron cosechadas en masa con base en su resistencia a roya amarilla y características agronómicas, proceso que se repitió hasta la generación $\mathrm{F}_{4}$. En la $\mathrm{F}_{5}$ se seleccionaron cuatro plantas que generaron cuatro líneas en $\mathrm{F}_{6}$.

Similarmente, en el caso de la tercera retrocruza $\left(\mathrm{BC}_{3}\right)$, en la $\mathrm{F}_{2}$ se seleccionaron 246 plantas que se avanzaron en homocigosis a través de familias por surco y se derivaron 246 líneas $\mathrm{F}_{5}$; así mismo, en esta retrocruza en la $\mathrm{F}_{2}$ se seleccionó, con base en su resistencia a roya amarilla y características agronómicas, un número indefinido de plantas que se cosecharon en masa para derivar cuatro líneas en $\mathrm{F}_{5}$.

\section{Evaluación de la enfermedad}

Las líneas obtenidas y los progenitores se evaluaron en etapa de planta adulta durante el ciclo primavera-verano 2017 en Chapingo, México, en el Campo Experimental Valle de México del Instituto Nacional de Investigaciones Forestales, Agrícolas y Pecuarias (INIFAP-CEVAMEX), bajo incidencia natural de roya amarilla y se evaluó la severidad con una escala de 0 a 100 (Roelfs et al., 1992).

Se evaluó la resistencia en plántula de las familias en $F_{5}$ y $F_{6}$ en el Laboratorio Nacional de Royas y Otras Enfermedades del Cereales (LANAREC) del INIFAPCEVAMEX, bajo condiciones de invernadero con dos repeticiones. Las familias se sembraron en charolas de plástico y 14 días después de siembra las plántulas se inocularon con una suspensión de urediniosporas del aislado de roya amarilla CMEX 14.25 , cuya fórmula de avirulencia/virulencia es Yr1, 5a, 5b, 10, 15, 24, 26, Poll/Yr2, $3,6,7,8,9,17,27,37,32$ (Huerta-Espino et al., 2015). Las urediniosporas se suspendieron en aceite mineral $\left(\right.$ Soltrol $\left.^{\circledR}\right)$ y fueron asperjadas con un atomizador conectado a un compresor eléctrico. Las plántulas inoculadas se colocaron en una cámara de incubación a temperatura de $4^{\circ} \mathrm{C}$ por 24 h y rocío al $100 \%$ por 1 h; posteriormente, se trasladaron al invernadero y 15 días después de la inoculación se registró su reacción a la roya amarilla con la escala de 1 a 9 propuesta por McNeal et al. (1971), donde lecturas del 1 al 6 se consideran resistentes y del 7 al 9 susceptibles. En la evaluación se incluyeron BG515, Bor100 y genotipos diferenciales con Yr5a y Yr15 como testigos.

\section{Análisis molecular}

El análisis molecular se llevó a cabo en el laboratorio de biotecnología del programa de mejoramiento molecular de trigo en el Centro Internacional de Mejoramiento de Maíz y Trigo (CIMMYT). Para la extracción de ADN se recolectaron porciones de tejido foliar y se colocaron en tubos de $1.1 \mathrm{~mL}$ en placas de 96 pozos; posteriormente, se almacenaron a $-80{ }^{\circ} \mathrm{C}$ durante $3 \mathrm{~h}$, después se transfirieron a un liofilizador y mantuvieron a temperatura de $-50^{\circ} \mathrm{C}$ a un nivel de vacío de 0.0 a $0.120 \mathrm{mbar}$ por $48 \mathrm{~h}$. El tejido se molió colocando balines de $4 \mathrm{~mm}$ por 2 o 3 min hasta obtener polvo, se empleó un molino Geno Grinder® (Metuchen, NJ, EUA). La cuantificación y valoración de la calidad del ADN se realizó de acuerdo con los protocolos de laboratorio para trigo descritos por Dreisigacker et al. (2016). Para detectar la presencia de los genes se utilizaron los marcadores SNP (Single Nucleotide Polymorphism) Y5/SPKASP3 y Yr5New para el gen Yr5a (Marchal et al., 2018), Yr15-R5 y Yr15-R8 para el gen Yr15 (Ramirez-Gonzalez et al., 2015). Adicionalmente, se utilizaron los marcadores Ventriup +Ln STS (Sequence-Tagged Sites) y VPM_SNP (Helguera et al., 2003) para observar la frecuencia del gen Yr17.

En el caso de los marcadores SNP se utilizó la tecnología de genotipificación Kompetitive Allele Specific PCR genotyping system, donde se utilizaron muestras control FAM y VIC correspondientes a los alelos positivo y negativo, HET (heterocigotos) y muestra blanco (agua); se ejecutó con la temperatura de amplificación de acuerdo con las condiciones necesarias para cada marcador (Dreisigacker et al., 2016). La lectura de los productos de PCR se realizó en un lector de placas fluorescentes BMG Pherastar Plus (Alemania) y para la visualización geográfica de datos genotípicos se utilizó el software KlusterCallerTM. Se consideraron como positivos los datos genotípicos que presentaron el alelo favorable para resistencia a roya y como negativos a los datos genotípicos que no portaron el alelo. Para el marcador Ventriup + Ln del gen Yr17 se incluyeron como testigos Avocet (control negativo) y Milán (control positivo), las condiciones usadas para la amplificación fueron las reportadas por Dreisigacker et al. (2016). Las muestras se colocaron en gel de agarosa $2.0 \%$, se utilizó 1X TBE (Tris-Borato EDTA) para la preparación del gel y como buffer de electroforesis. Las 
bandas amplificadas se tiñeron con bromuro de etidio y se observaron en luz ultravioleta en un transiluminador BioDoc-It ${ }^{\text {TM }}$ (Upland, CA, EUA).

\section{RESULTADOS Y DISCUSIÓN}

Se obtuvieron 139 líneas a partir de la población derivada de dos retrocruzas $\left(\mathrm{BC}_{2}\right)$, de las cuales, en 135 no se aplicó ningún criterio de selección y cuatro se seleccionaron con base en criterios agronómicos deseables. De igual forma, de la población derivada de tres retrocruzas se obtuvieron 250 líneas donde 246 fueron sin selección y cuatro con selección (389 líneas en total).

De las 389 líneas evaluadas, 287 (74 \%) mostraron lecturas de 0 a $10 \%$ en la escala de severidad de la enfermedad; de éstas, 163 líneas mostraron $0 \%$ de infección, lo que equivale a $42 \%$ del total. Las restantes 102 líneas (26\%) tuvieron lecturas de severidad entre 15 y $70 \%$, esta última fue la lectura máxima registrada. La variedad Bor 100 registró lectura de 10 \% de infección, lo que sugirió la posibilidad de que las 163 líneas con severidad $0 \%$ pueden ser resultado de la introgresión del gen Yr5a, Yr15 o ambos. En estas 163 líneas se observaron fenotipos semejantes a Bor100, incluso algunos con expresiones fenotípicas superiores a dicho cultivar.

Los resultados de la evaluación en estado de plántula mostraron 300 líneas resistentes; es decir, con infección entre 0 y 6 . Los 89 restantes fueron susceptibles a la raza evaluada. BG515 mostró infección de 0, mientras que Bor100 tuvo 6. La raza CMEX14.25 es avirulenta a los genes Yr1, 5a, 5b, 10, 15, 24 y Poll (Huerta-Espino et al., 2015). Fenotípicamente no se pueden distinguir las líneas portadoras de Yr5a de otras portadoras de Yr15 u otro gen de resistencia como Yr5b (YrSP) o en combinación cuando existe más de un gen y el gen de mayor efecto es epistático (Huerta-Espino et al., 2003); además, en México no se han identificado razas de roya amarilla virulentas a Yr5a y a Yr15; por lo anterior, se utilizaron marcadores moleculares para identificar las líneas portadoras de Yr5a y Yr15 de forma individual o en conjunto.

Los marcadores moleculares asociados a los genes Yr5a, 15 y 17 permitieron identificar líneas con los genes de resistencia a roya amarilla en sus diferentes combinaciones (Cuadro 1). Se encontró que 23 líneas fueron negativas para los tres genes, 11 provienen de tres retrocruzas y 12 a partir de dos retrocruzas, todas ellas perteneciente al grupo donde no se realizó selección.

De las 246 líneas generadas a partir de tres retrocruzas sin selección, una fue positiva sólo a Yr5a y dos positivas sólo a Yr15. Las líneas positivas únicamente al gen Yr17 son las que se encontraron en mayor cantidad (168), lo que representa $68.3 \%$ del total de líneas de este grupo. No se encontraron líneas con la combinación Yr5a+Yr15, pero sí hubo ocho con Yr5a+Yr17. El segundo grupo con mayor cantidad estuvo conformado por las 39 líneas positivas a Yr15+Yr17, las cuales representan $15.9 \%$. El gen que se encontró el mayor número de veces en estado heterocigótico fue Yr75, 10 líneas en combinación con Yr 17 y una negativa para Yr5a y Yr17. También se encontraron tres líneas con HetYr5a y Yr17 en estado homocigótico en forma conjunta. Las tres líneas restantes fueron HetYr17 y negativas para Yr5a y Yr15.

Al realizar dos retrocruzas se obtuvieron líneas con más combinaciones de genes. En este grupo, nuevamente, la mayor cantidad fueron positivas para Yr17 (48 líneas); sin embargo, no hubo líneas positivas solo a Yr5a y cuatro fueron positivas sólo a Yr15. La segunda combinación más frecuente fue $Y r 15+Y r 17$ con 22 líneas, que representaron el $16.3 \%$, seguida de Yr5a+Yr17 con 14 líneas. La combinación de HetYr5a+Yr17 se encontró 12 veces, mientras que siete líneas sólo tuvieron el gen HetYr5a. Las combinaciones HetYr15+Yr17 y HetYr $17+$ Yr15 se encontraron en cinco y tres líneas, respectivamente. La combinación Yr5a+Yr15+Yr17 se encontró en dos líneas, otras dos HetYr17 y negativas para los otros dos genes. Solamente se encontró una línea para cada una de las siguientes combinaciones: Yr5a+Yr15, HetYr5a+Yr15+Yr17, HetYr5a+HetYr17 y HetYr5a+HetYr15+Yr17.

De las ocho líneas derivadas de la selección agronómica, cuatro de ellas a partir de tres retrocruzas fueron positivas para Yr15+Yr17; también se encontró esta combinación en dos líneas provenientes de dos retrocruzas. Las dos líneas restantes fueron positivas para los tres genes y la combinación HetYr15+Yr17, respectivamente.

Se calcularon los coeficientes de correlación de Pearson para los dos marcadores asociados a cada gen y se obtuvieron valores de $r=0.80 * *$ para los marcadores Yr15-R5 y Yr15-R8 usados en la detección de Yr15; $r=$ 0.89** para Y5/SPKASP3K y Yr5New usados para Yr5a; $r=0.83^{* *}$ para Ventriup + Ln y VPM_SNP. En el caso de los marcadores asociados a Yr17, se prefiere VPM_SNP, ya que ofrece la ventaja sobre Ventriup $+L n$ de detectar heterocigotos.

Las líneas de mayor interés en este estudio son aquellas que poseen los genes Yr5a y Yr 15, y corresponden a las entradas 358,368 y 395 (Cuadro 2); sin embargo, las entradas 297 y 344 presentan uno o dos de los genes en estado heterocigótico, lo que fenotípicamente se observa como resistente desde plántula por ser genes mayores y dominantes (GerechterAmitai et al., 1989; Marchal et al., 2018). 
Cuadro 1. Número y porcentaje de líneas con/sin selección positivas a los genes evaluados y sus combinaciones provenientes de dos y tres retrocruzas.

\begin{tabular}{|c|c|c|c|c|c|c|c|c|}
\hline \multirow[b]{2}{*}{ Gen/combinación } & \multicolumn{4}{|c|}{ Sin selección } & \multicolumn{4}{|c|}{ Con selección } \\
\hline & $\begin{array}{c}\mathrm{BOR} * 3 / \\
\mathrm{BG} 515 \\
\end{array}$ & $\%$ & $\begin{array}{l}\mathrm{BOR} * 2 / \\
\mathrm{BG} 515 \\
\end{array}$ & $\%$ & $\begin{array}{l}\mathrm{BOR} \star 3 / \\
\mathrm{BG} 515 \\
\end{array}$ & $\%$ & $\begin{array}{l}\mathrm{BOR} * 2 / \\
\mathrm{BG} 515 \\
\end{array}$ & $\%$ \\
\hline Ninguno & 11 & 4.5 & 12 & 8.9 & - & - & - & - \\
\hline Yr5a & 1 & 0.4 & - & - & - & - & - & - \\
\hline Yr15 & 2 & 0.8 & 4 & 3.0 & - & - & - & - \\
\hline Yr17 & 168 & 68.3 & 48 & 35.6 & - & - & - & - \\
\hline Yr5a+Yr15 & - & - & 1 & 0.7 & - & - & - & - \\
\hline Yr5a+Yr17 & 8 & 3.3 & 14 & 10.4 & - & - & - & - \\
\hline Yr15+Yr17 & 39 & 15.9 & 22 & 16.3 & 4 & 100 & 2 & 50 \\
\hline Yr5a+Yr15+Yr17 & - & - & 2 & 1.5 & - & - & 1 & 25 \\
\hline HetYr5a & - & - & 7 & 5.2 & - & - & - & - \\
\hline HetYr15 & 1 & 0.4 & - & 0.0 & - & - & - & - \\
\hline HetYr17 & 3 & 1.2 & 2 & 1.5 & - & - & - & - \\
\hline HetYr5a+Yr17 & 3 & 1.2 & 12 & 8.9 & - & - & - & - \\
\hline HetYr5a+Yr15+Yr17 & - & - & 1 & 0.7 & - & - & - & - \\
\hline HetYr15+Yr17 & 10 & 4.1 & 5 & 3.7 & - & - & 1 & 25 \\
\hline HetYr17+Yr15 & - & - & 3 & 2.2 & - & - & - & - \\
\hline HetYr5a+HetYr17 & - & - & 1 & 0.7 & - & - & - & - \\
\hline HetYr5a+HetYr15+Yr17 & - & - & 1 & 0.7 & - & - & - & - \\
\hline Total & 246 & 100 & 135 & 100 & 4 & 100 & 4 & 100 \\
\hline
\end{tabular}

Het: heterocigoto

Cuadro 2. Resultados de análisis molecular de los progenitores, líneas positivas a Yr5a+Yr15 o Yr17 y respuesta a roya amarilla causada por Puccinia striiformis $f$. sp. tritici en plántula y planta adulta.

\begin{tabular}{|c|c|c|c|c|c|c|c|c|c|}
\hline \multirow{2}{*}{ No. } & \multirow{2}{*}{ Genotipo } & \multicolumn{8}{|c|}{ Marcadores moleculares } \\
\hline & & Yr15-R5 & Yr15-R8 & Y5/SPKASP3 & Yr5New & VPM_SNP & Ventriup $+\mathrm{Ln}$ & Sev $P$ & SevPA \\
\hline 388 & Blanca Grande 515 & Yr15+ & Yr15+ & Het & Het & VPM- & Yr17- & 0 & 0 \\
\hline 390 & Borlaug 100 & Yr15- & Yr15- & Yr5a- & Yr5a- & VPM+ & Yr17+ & 6 & 10 \\
\hline 297 & Bor100*2/BGde515.242 & Het & Het & Het & Het & VPM+ & Yr17+ & 0 & 0 \\
\hline 344 & Bor100*2/BGde515.278 & Yr15+ & Yr15+ & Het & Het & VPM+ & Yr17+ & 0 & 0 \\
\hline 358 & Bor100*2/BGde515.291 & Yr15+ & Yr15+ & Yr5a+ & Yr5a+ & VPM- & Yr17- & 0 & 0 \\
\hline 368 & Bor100*2/BGde515.299 & Yr15+ & Yr15+ & Yr5a+ & Yr5a+ & VPM+ & Yr17+ & 0 & 0 \\
\hline 395 & Bor100*2/BGde515(468) & Yr15+ & Yr15+ & Yr5a+ & Yr5a+ & VPM+ & Yr17+ & 0 & 0 \\
\hline
\end{tabular}

Sev P. \% de severidad en plántula; Sev PA: \% de severidad en planta adulta. 
Se encontró que Bor100 posee el gen Yr17, lo que justifica el gran porcentaje de líneas positivas para este gen y gran parte de su genoma es recuperado al hacer las retrocruzas. Genotipos con Yr17 se han utilizado en muchos programas de mejoramiento de trigo en Europa. La virulencia para este gen se encontró desde 1994 en Inglaterra y se extendió por el norte y oeste de Europa en los años siguientes (Chen and Kang, 2017). Actualmente, aislados virulentos para este gen están ampliamente distribuidos en el mundo (Rodríguez et al., 2009; SharmaPoudyal et al., 2013; Wellings et al., 2009); no obstante, en México la raza virulenta a Yr17 es avirulenta a Yr27 y Yr31 y muestra virulencia parcial en planta adulta, por lo que Borlaug 100 en planta adulta es moderadamente resistente debido a que los aislados presentes en México son parcialmente virulentos al gen Yr17, además de la presencia de los genes de raza no especifica Yr29 y Yr30, entre otros no identificados. El gen Yr17 aún puede proporcionar resistencia contra varias razas de roya amarilla si se usa en combinación con otros genes (Iqbal et al., 2016), como en el caso del presente estudio donde está en combinación con el Yr5a, Yr15 o ambos.

A pesar de que Jackson (2011) reportó que BG515 posee Yr5a y Yr15 en condición homocigótica, en este estudio se encontró que Yr5a está en forma heterocigótica (Cuadro 2), razón por la cual el número de líneas positivas a este gen fue bajo en comparación con las positivas a Yr15 (Cuadro 1), además de que no se utilizaron marcadores para detectar Yr5a en la etapa de retrocruzas. El número de combinaciones de genes Yr5a y Yr15 encontradas fue menor cuando se realizó una tercera retrocruza, incluso cuando se realizó selección de las cuatro líneas que mostraron la misma combinación de genes. Se ha observado que una retrocruza es suficiente para obtener líneas que posean genes de resistencia de sus progenitores donadores sin afectar el rendimiento e incluso aumentar su potencial (Singh y Huerta-Espino, 2004). Lo anterior se confirmó en este estudio, pues cuando se realizaron tres retrocruzas la variabilidad se redujo y no se encontraron líneas positivas a los dos genes de interés (Cuadro 1).

En una cruza simple, para incorporar dos genes dominantes, la frecuencia de familias es de 15:1 (resistentes:susceptibles), de la cual se obtienen los genotipos $A_{-} B_{-}, A \_b b, a a B_{-}$, aabb $\left(A=Y r 5 a^{+}, a=Y r 5 a^{-} ; B=\right.$ $\left.Y_{r 15^{+}}, b=Y_{r} 15^{-}\right)$en proporción 9:3:3:1. En $\mathrm{BC}_{1}$ la proporción de familias es 1:1:1:1, mientras que en $\mathrm{BC}_{2}$ la proporción es 1:3:3:9, donde las familias de interés (A_B_) se encuentran en menor proporción, lo que disminuye la probabilidad de tener Yr5a y Yr15 en conjunto de forma positiva; lo anterior sucede nuevamente en $\mathrm{BC}_{3}$, ya que la proporción es 1:7:7:49.
En este estudio se encontró que la variedad BG515 presentó Yr5a en forma heterocigótica, por lo que la cruza inicial fue $\mathrm{AaBB} \times$ aabb y la $\mathrm{F}_{1}$ estuvo conformada por los genotipos AaBb y aaBb (1:1); al realizar una, dos y tres retrocruzas las proporciones son 1:1:3:3, 1:3:7:21 y 1:7:15:105, respectivamente (para los genotipos A_B_, A_bb, aaB_ y aabb), de tal forma que al avanzar en las retrocruzas la proporción de las familias con ambos genes positivos fue disminuyendo, de ahí la importancia de la selección asistida por marcadores moleculares; sin embargo, los marcadores a utilizar deben ser marcadores de diagnóstico, de lo contrario se corre el riesgo de perder los genes de resistencia en el proceso de selección o terminar con plantas positivas en baja frecuencia como en el presente estudio. Al tomar en cuenta el gen Yr77 proveniente de Bor100, la frecuencia del mismo aumentó conforme se avanzó en las retrocruzas debido a la recuperación de genoma del cultivar.

Se encontraron familias que mostraron resistencia en la evaluación en plántula, pero fueron negativas a los marcadores, posiblemente debido a la presencia de otro gen que le confiere resistencia a la raza evaluada; además, hubo casos en que los marcadores indicaron positivo para alguno de los dos genes, pero fueron susceptibles en plántula, quizá debido a mezclas de semilla durante la siembra.

La combinación de múltiples genes de resistencia efectivos en todas las etapas en una sola variedad es la mejor estrategia para una resistencia duradera a royas (Hulbert y Pumphrey, 2014), por lo que las líneas avanzadas identificadas en el presente estudio que poseen los genes Yr5a, Yr15 y Yr 77 pueden ser utilizadas en los programas de mejoramiento de trigo en México como progenitoras para el control genético de la roya amarilla; por otra parte, estas líneas genotípicamente tienen el 86 \% (dos retrocruzas) del fondo genético de la variedad elite recurrente (Borlaug 100), incluso se observó que algunas líneas mostraron mejor expresión fenotípica para rendimiento de grano, por lo que serán probadas en ensayos de rendimiento en el programa de trigo del INIFAP para su futura liberación como nuevos cultivares, lo que demuestra que la metodología de la retrocruzas con la selección asistida con marcadores moleculares y selección fenotípica hacia homocigosis es una estrategia importante para mejorar la resistencia y caracteres agronómicos.

\section{CONCLUSIONES}

Se identificaron tres familias avanzadas con las características de Borlaug 100 que poseen los genes Yr5a y Yr15 en condición homocigótica. Se identificó una familia semejante a Blanca Grande 515 (Yr5a heterocigoto, Yr15 
homocigoto) y una última con ambos genes en condición heterocigótica.

\section{BIBLIOGRAFÍA}

Acquaah G. (2012) Principles of Plant Genetics and Breeding. Second edition.: John Wiley and Sons. Oxford, UK. 628 p.

Ali S., J. Rodriguez-Algaba, T. Thach, C. K. Sørensen, J. G. Hansen, P. Lassen, ... and M. S. Hovmøller (2017) Yellow rust epidemics worldwide were caused by pathogen races from divergent genetic lineages. Frontiers in Plant Science 8:1057, https://doi.org/10.3389/fpls.2017.01057

Begum S., M. Iqbal, I. Ahmed, M. Fayyaz, A. Shahzad and G. M. Ali (2014) Allelic variation at loci controlling stripe rust resistance in spring wheat. Journal of Genetics 93:579586, https://doi.org/10.1007/s12041-014-0413-9

Camacho C. M. A., G. Chávez V., G. Fuentes D., P. Figueroa L., J. Huerta E. y H. E. Villaseñor M. (2017) Borlaug 100: Variedad de trigo harinero para el noroeste de México. Folleto Técnico No. 100. Campo Experimental Norman E. Borlaug. Instituto Nacional De Investigaciones Forestales, Agrícolas y Pecuarias. Cd. Obregón, Sonora, México. $32 \mathrm{p}$.

Chen X. and Z. Kang (2017) Stripe rust resistance. In: Stripe Rust. X. Chen and Z. Kang (eds.). Springer. Dordrecht, The Netherlands. pp:353-558, https://doi.org/10.1007/978-94-024-1111-9_5

Díaz C. H. L., S. G. Leyva M., H. E. Villaseñor M., M. Vargas H., R. Hortelano S. R., Y. R. Valdez R. y E. Martínez C. (2018) Control químico de la roya lineal en diferentes etapas de desarrollo del trigo en Terrenate Tlaxcala. Revista Mexicana de Ciencias Agrícolas 9:1067-1074. https://doi.org/10.29312/remexca.v9i5.1510

Dreisigacker S., D. Sehgal, A. E. Reyes J., B. Luna G., S. Muñoz Z., C. Núñez R., ... and S. Mall (2016) CIMMYT Wheat Molecular Genetics: Laboratory Protocols and Applications to Wheat Breeding CIMMYT. Mexico, D. F. 142 p.

Gerechter-Amitai Z. K., C. H. van Silfhout, A. Grama and F. Kleitman (1989) Yr15 - a new gene for resistance to Puccinia striiformis in Triticum dicoccoides sel. G-25. Euphytica 43:187-190, https://doi.org/10.1007/BF00037912

GRIS, Genetic Resources Information System for Wheat and Triticale (2017) Genetic Resources Information System for Wheat and Triticale. CIMMYT. Mexico City. http://www.wheatpedigree.net/sort/ show/111755 (October 2019)

Helguera M., I. A. Khan, J. Kolmer, D. Lijavetzky, L. Zhong-Qi and J. Dubcovsky (2003) PCR assays for the Lr37-Yr77-Sr38 cluster of rust resistance genes and their use to develop isogenic hard red spring wheat lines. Crop Science 43:1839-1847 https://doi.org/10.2135/cropsci2003.1839

Hubbard A., C. M. Lewis, K. Yoshida, R. H. Ramirez-Gonzalez, C. Vallavieill-Pope, J. Thomas ... and D. Saunders (2015) Field pathogenomics reveals the emergence of a diverse wheat yellow rust population. Genome Biology 16:23, https://doi.org/10.1186/s13059-015-0590-8

Huerta-Espino J. and R. P. Singh (2017) First detection of virulence in Puccinia striiformis f. sp. tritici to wheat resistance genes Yr10 and Yr24 (=Yr26) in Mexico. Plant Disease 101:1676, https://doi.org/10.1094/PDIS-04-17-0532-PDN

Huerta-Espino J., R. P. Singh, H. E. Villaseñor-Mir, E. Espitia-Rangel y S. G. Leyva-Mir (2003) Postulación de genes de resistencia a la roya de la hoja (Puccinia triticina Ericks.) en plántula y planta adulta en genotipos élite de trigo harinero (Triticum aestivum L.) Revista Mexicana de Fitopatología 21:239-247.

Huerta-Espino J., H. E. Villaseñor-Mir, M. F. Rodríguez-García and R. P. Singh (2015) Emerging new virulence gene combinations in the Mexican Pst population. In: Borlaug Global Rust Initiative Technical Workshop. September 17-20. Sydney, Australia. pp:16.

Hulbert S. and M. Pumphrey (2014) A time for more booms and fewer busts? Unraveling cereal-rust interactions. Molecular Plant-Microbe Interactions 27:207-214 https://doi.org/10.1094/MPMI-09-13-0295-FI

Iqbal M., M. Ejaz, I. Ahmed, A. Shahzad and G. M. Ali (2016) Molecular genetic variation for stripe rust resistance in spring wheat. Pakistan Journal of Agricultural Sciences 53143-150, https://doi.org/10.21162/PAKJAS/16.2503
Jackson L. (2011) Wheat cultivars for California. University of California, Davis. Davis, California. 69 p. http://smallgrains.ucdavis.edu/ cereal_files/WhtCVDescLJ11.pdf (September 2019).

Macer R C. F. (1966) The formal and monosomic genetic analysis of stripe rust (Puccinia striiformis) resistance in wheat. In: Proceedings of the Second International Wheat Genetics Symposium. J. Mackey (ed.) Lund University. Lund, Sweden. pp:127-142.

Marchal C., J. Zhang, P. Zhang, P. Fenwick, B. Steuernagel, N. M. Adamski, ... and C. Uauy (2018) BED-domain-containing immune receptors confer diverse resistance spectra to yellow rust. Nature Plants 4:662-668, https://doi.org/10.1038/s41477-018-0236-4

McIntosh R. A., J. Dubcovsky, W. J. Rogers, C. Morris and X. C. Xia (2017) Catalogue of gene symbols for wheat: 2017 supplement KOMUGI - Wheat Genetic Resources Database. National BioResource Project. Shigen, Japan. https://shigen.nig.ac.jp/ wheat/komugi/genes/macgene/supplement2017.pdf (August 2020).

McNeal F. H., C. F. Konzak, E. P. Smith, W. S. Tate and T. S. Russell (1971) A uniform system for recording and processing cereal research data. Agricultural Research Service Bulletin 34:121-143.

Nagarajan S., S. K. Nayar and P. Bahadur (1986) Race 13 (67S8) of Puccinia striiformis virulent on Triticum spelta var. album in India. Plant Disease 70:173, https://doi.org/10.1094/PD-70-173d

Naruoka Y., K. Ando, P. Bulli, K. T. Muleta, S. Rynearson and M. O. Pumphrey (2016) Identification and validation of SNP markers linked to the stripe rust resistance gene Yr5 in wheat. Crop Science 56:3055 3065, https://doi.org/10.2135/cropsci2016.03.0189

Ramirez-Gonzalez R. H., V. Segovia, N. Bird, P. Fenwick, S. Holdgate, S. Berry, ... and C. Uauy (2015) RNA-Seq bulked segregant analysis enables the identification of high-resolution genetic markers for breeding in hexaploid wheat. Plant Biotechnology Journal 13:613-624, https://doi.org/10.1111/pbi.12281

Rodríguez G. M. F., J. Huerta E., H. E. Villaseñor M. y E. Solís M. (2009) Virulencia de la roya amarilla del trigo en las principales zonas productoras de riego en México. Agricultura Técnica en México 35:179-187.

Rodríguez-García M. F., J. Huerta-Espino, H. E. Villaseñor-Mir, J. S. Sandoval I. y R. P. Singh (2010) Análisis de virulencia de la roya amarilla (Puccinia striiformis f. sp. tritici) del trigo (Triticum aestivum L.) en los Valles Altos de México. Agrociencia 44:491-502.

Roelfs A. P., R. P. Singh y E. E. Saari (1992) Las Royas del Trigo: Conceptos y Métodos para el Manejo de esas Enfermedades. CIMMYT. México, D. F. 81 p.

Sharma-Poudyal D., X. M. Chen, A. M. Wan, G. M. Zhan, Z. S. Kang, S. Q. Cao, ... and L. J. Patzek (2013) Virulence characterization of international collections of the wheat stripe rust pathogen Puccinia striiformis f. sp. tritici. Plant Disease 97:379-386, https://doi.org/10.1094/PDIS-01-12-0078-RE

Singh R. P. and J. Huerta-Espino (2004) The use of 'single-backcross, selected-bulk' breeding approach for transferring minor genes based rust resistance into adapted cultivars. In: Proceedings of $54^{\text {th }}$ Australian Cereal Chemistry Conference and $11^{\text {th }}$ Wheat Breeders Assembly. C. K. Black, J. F. Panozzo and G. J. Rebetzke (eds). Cereal Chemistry Division, Royal Australian Chemical Institute. Canberra, Australia. pp:48-51.

Singh R. P., J. Huerta-Espino and A. P. Roelfs (2002) The wheat rusts. In Bread Wheat: Improvement and Production. B. C. Curtis, S Rajaram, H. Gomez MacPherson (eds.). Food and Agriculture Organization of the United Nations. Rome. pp:227-249.

Solís M. E., J. Huerta E., P. Pérez H., H. E. Villaseñor M., A. Ramírez R. y M. L. de la Cruz G. (2016) Alondra F2014, nueva variedad de trigo harinero para el Bajío, México. Revista Mexicana de Ciencias Agrícolas 7:1225-1229, https://doi.org/10.29312/remexca.v7i5.248

Vagndorf N., P. S. Kristensen, J. R. Andersen, A. Jahoor and J. Orabi (2018) Marker-assisted breeding in wheat. In: Next Generation Plant Breeding. Y. Ozden Çiftçi (ed.). IntechOpen, London, UK. pp:422, https://doi.org/10.5772/intechopen.74724

Villaseñor M. H. E., E. Espitia R., J. Huerta E., E. Solís M., J. Ireta M., L. Osorio A. y P. Pérez H. (2014) Nana F2007, cultivar de trigo para siembras de temporal en México. Revista Mexicana de Ciencias Agrícolas 5:1363-1368, https://doi.org/10.29312/remexca.v0i7.1124

Wellings C. R. (2011) Global status of stripe rust: a review of 
historical and current threats. Euphytica 179:129-141, https://doi.org/10.1007/s10681-011-0360-y

Wellings C. R., R. P. Singh, A. Yahyaoui, K. Nazari and R. A. McIntosh (2009) The development and application of near-isogenic lines for monitoring cereal rust pathogens. In: Proceedings of Oral Papers. 2009 Technical Workshop. Borlaug Global Rust Initiative. Cd. Obregón, Sonora, México. pp:77-87. 